\title{
Bit-pairing Codification for Binary Pattern Projection System
}

\author{
Jun Cheng, Ronald Chung \\ Dept. of ACAE \\ The Chinese Univ. of Hong Kong \\ Hong Kong, CHINA \\ jcheng,rchung@acae.cuhk.edu.hk
}

\author{
Edmund Y. Lam \\ Dept. of EEE \\ The Univ. of Hong Kong \\ Hong Kong, CHINA \\ elam@eee.hku.hk
}

\author{
Kenneth S.M. Fung \\ ASM assembly automation Ltd. \\ Hong Kong, CHINA \\ smfung@asmpt.com
}

\begin{abstract}
In a previous work, we proposed a new binary-light projection mechanism that had a much reduced system size that made it particularly suitable for $3 D$ shape inspection of semiconductor products. The inspection speed of the mechanism was governed by the number of required images which also equaled the number of shiftings of the grating. In this paper we address how inspection speed could be gained, i.e., how the number of required images could be reduced, by the incorporation of two neighboring bits in the codification of each scene element. We provide an optimal design of such a codification strategy. A solution to the shifting strategy optimization is also proposed that is applicable to any given binary patterns. Theoretical analysis and real image experiments are presented to illustrate the workability of the solutions. ${ }^{1}$
\end{abstract}

\section{Introduction}

In advanced electronic manufacturing that involves say die-to-die bonding, microscopic surfaces like solder bumps on wafers have to be inspected in 3D. There have been a few gray-level coding-based approaches proposed for 3D reconstruction, but they all suffer from problems of image noise and gray level saturation $[4,5,7]$. One way to counteract image noise and gray level saturation and alike problems is to replace the analog signals by discrete ones like binary signals. Binary pattern has been explored under the name of structured light-based 3D reconstruction. In $[1,2,8]$, the authors proposed the use of a pattern that consisted of a dot matrix of $n \times n$ binary light beams. By projecting a sequence of $n$ patterns, they could encode $2^{n}$ stripes. This en-

\footnotetext{
${ }^{1}$ The work described in this paper was substantially supported by a grant from the Innovation and Technology Commission of Hong Kong Special Administrative Region, China, under an Innovation and Technology Fund with Project Code UIM/111.
}

coding mechanism is furthered of changing the binary codification to a Gray codification[6].

Yet, in traditional binary pattern projection the illumination pattern was meant to be an array of on-off controllable pattern. Such arrangement is however not suitable for the inspection of the targeted devices (e.g., wafer bumps). The reason is that such devices have large physical size, which is undesirable in semiconductor inspection.

In a previous work [3], we proposed an alternative binary pattern projection mechanism that was adapted to own a small size. The mechanism consisted of only a single light source in combination with a binary grating for projecting binary pattern. The binary illumination was to be varied by shifting the binary grating in space relative to the single light source.

The inspection speed of the new mechanism was governed by the number of required images which also equaled the number of shiftings of the grating. Suppose there were $M$ alternative fringes on the grating. Before any shifting of the grating took place, these $M$ fringes together with the single light source corresponded to $M$ scene elements in $3 \mathrm{D}$. Shifting the binary grating $n$ times in space would then produce a number of $n$-bit long codewords, one for each of the $M$ scene elements. To give a unique codeword to each of the $M$ scene elements the number $n$ of grating shiftings must exceed a certain bound; in fact the lower bound of $n$ was $\log _{2} M$.

What was challenging in the new mechanism was, the bit values ( 1 or 0$)$ of different positions of the grating at any particular time were globally related to one another for the fact that the binary grating was constant, the light source was only one, and the change in pattern value was only induced by a physical and global shifting of the grating in space. Such a correlation between the codewords of different scene elements was the price to pay for the small size of the inspection system, and it generally made $n$ much greater than the lower bound $\log _{2} M$ for unique codeword for each of the scene elements.

In this paper we address how $n$ - the number of images 
(i.e., the number of grating shiftings) for inducing unique codeword for each of the scene elements - could be reduced, resulting in a gain in the inspection speed.

We propose that every two spatially adjacent bits are grouped together to produce the codeword for the corresponding scene element in space, thereby enriching the information in the codewords, and in the end reducing the total number of images needed. Such a mechanism, which we refer to as the bit-pairing codification mechanism, allows $n$ shiftings of the grating (of $M$ alternate fringes) to produce $2 n$-bit long codewords, one for each of the $M$ corresponding scene elements. The codewords have more information content and are thus more distinct and more likely to be unique for the same number of captured images.

There is of course a price to pay for the bit-pairing codification scheme: some of the very sharp discontinuities in the target shape could be smoothed out in the final 3D recovery. However, we figure that in many practical circumstances inspection speed is of an importance that could well justify that particular expense, and this work is of immense value.

\section{Shifting strategy optimization algorithm}

Given a fixed binary grating in our system, adopting different shifting strategies will produce different codeword sets with different lengths. In order to reconstruct 3D surface with high speed, the shifting strategy should be optimized.

\subsection{Traditional codification mechanism}

In the proposed system, the codeword of every point is formed by shifting one single periodical binary grating. Shifting a grating of period $P$ several times is equivalent to dissecting the grating into several stripes which we refer to as the Grating-Motion Induced Zone (GZ). The more the shifting times, the higher the column resolution will be. The shifting displacement $\triangle P$ in each shifting is actually determined by the requirement of the column resolution which has a direct impact on the resolution of the 3D recovery.

Suppose the required resolution is such that each period of the grating are to be dissected into $M \mathrm{GZs}$ :

$$
M=\operatorname{int}\left(\frac{P}{\triangle P}\right)
$$

among which $M / 2$ GZs are ' 0 ' and $M / 2$ GZs are ' 1 '.

To assign unique codeword to these $M$ GZs, $M / 2$ patterns should be projected at least. Therefore, the formed codewords will have $M / 2$ bits. However, among these codewords formed by $M / 2$ patterns, there is a codeword whose bits are all 0 . The codeword of all 0's is not usable because with its use one could not distinguish between positions covered by the pattern projection and those that are occluded. To avoid the problem, an additional shifting is performed to make sure that all codewords that are used should have at least one bit being ' 1 '. Fig. 1(a) illustrates the codeword of a particular position on the grating. In this example, one period of the pattern comprises $10 \mathrm{GZs}$. Therefore, 6 patterns should be projected to form codeword. The codeword of the first GZ is 011111.

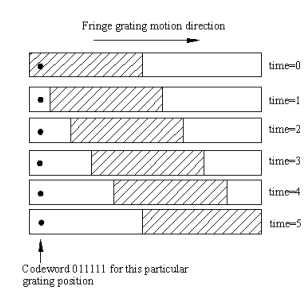

(a) Traditional codification

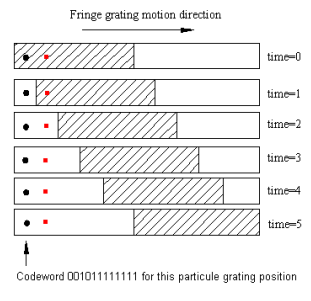

(b) Bit-pairing codification

\section{Figure 1. Illustration of traditional and bit- pairing codification mechanism.}

\subsection{Bit-pairing codification mechanism}

The pattern design can further be optimized by using a bit-pairing mechanism. Every two neighbor bits can be grouped together to produce codeword. Once a bit-pairing mechanism is adopted, shifting the M-bit binary pattern $n$ times will produce $\mathrm{M} 2 \mathrm{n}$-bit-length codewords. The length of these codewords is $2 \mathrm{n}$ bits, which is longer than that produced without a bit-pairing mechanism. Therefore, the longer length codeword means a greater likelihood that these $\mathrm{M}$ codewords are unique. So adopting bit-pairing mechanism could reduce the number of images. Fig. 1(b) illustrates the bit-pairing codification mechanism. With the same 6 patterns being projected, 12-bit-length codeword of that particular position can be produced, which is double length of that of traditional produced codeword. The codeword of the first GZ is 001011111111.

\subsection{Optimization algorithm}

The objective of shifting strategy optimization is to select minimal number of shiftings such that the codewords combined by these shiftings are unique. If the difference between two codewords is nonzero, then these two codewords are different. Therefore, the difference matrix formed by differences between any two codewords can help to judge whether all the codewords are unique or not. Once all the columns of the difference matrix have at least one element whose value is nonzero, the codewords will be unique. 
Given an arbitrary pattern $\mathrm{P}$ that comprises $n \mathrm{GZs}$,

$$
P=C_{1} C_{2} \cdots C_{n}
$$

where $C_{i} \in\{0,1\}, i=1,2, \cdots, n$. There at most is $n$ different shifting which is equivalent to the number of GZs in one period.

$$
\left\{\begin{array}{l}
P_{1}=C_{1} C_{2} \cdots C_{n-1} C_{n} \\
P_{2}=C_{n} C_{1} \cdots C_{n-2} C_{n-1} \\
\vdots \\
P_{n}=C_{2} C_{3} \cdots C_{n} C_{1}
\end{array}\right.
$$

Suppose one bit and the bit on its immediate right are to comprise the bit-pairing. In Equation 2, every two columns will be a bit-pairing. Convert every two adjacent columns $k$ th and $(k+1)$ th $(k=1,2, \cdots, n)$ columns into a new column $T_{k}$. Then the new matrix $T$ converted from matrix $P$ will be,

$$
\left\{\begin{array}{l}
T_{1}=T_{1,1} T_{1,2} \cdots T_{1, n-1} T_{1, n} \\
T_{2}=T_{2,1} T_{2,2} \cdots T_{2, n-1} T_{2, n} \\
\vdots \\
T_{n}=T_{n, 1} T_{n, 2} \cdots T_{n, n-1} T_{n, n}
\end{array}\right.
$$

Where $T_{i, j}=2 * P_{i, j}+P_{i, j+1}(j=1, \cdots, n-1), T_{i, n}=$ $2 * P_{i, n}+P_{i, 1}$ and $T_{i j} \in\{0,1,2,3\}$.

Among these $n$ shiftings, adopting different shifting strategies will lead to different image number, i.e. different sets of codeword. The objective of shifting strategy optimization is to select a number of pattern shifting to form unique codeword with minimal bits. The difference matrix formed by differences between any two codewords can help to judge whether all the codewords are unique or not. Once all the columns of the difference matrix have at least one element whose value is nonzero, the codewords will be unique. Thus, the optimization problem can be converted to select minimal number of rows from the difference matrix so that all the columns have at least one element whose value is nonzero.

Construct a matrix $E$ from matrix $T$. Every column subtracts all the other columns. Thus a new matrix $E$ can be obtained, whose size is $n \times l, l=C_{n}^{2}$ and elements are absolute values of difference. Because of $T_{i j} \in\{0,1,2,3\}$, the difference of any two elements in matrix $T$ will be in $\{-3,-2,-1,0,1,2,3\}$. ' 0 ' represents that the two elements are the same and nonzero represents that the two elements are different.

$$
E=\left(\begin{array}{cccc}
e_{11} & e_{12} & \cdots & e_{1 l} \\
e_{21} & e_{22} & \cdots & e_{2 l} \\
\vdots & & & \\
e_{n 1} & e_{n 2} & \cdots & e_{n l}
\end{array}\right)=
$$

$$
\left(\begin{array}{ccccc}
\left|T_{1,1}-T_{1,2}\right| & \cdots & \left|T_{1,1}-T_{1, n}\right| & \cdots & \left|T_{1, n-1}-T_{1, n}\right| \\
\left|T_{2,1}-T_{2,2}\right| & \cdots & \left|T_{2,1}-T_{2, n}\right| & \cdots & \left|T_{2, n-1}-T_{2, n}\right| \\
\vdots & & & & \\
\left|T_{n, 1}-T_{n, 2}\right| & \cdots & \left|T_{n, 1}-T_{n, n}\right| & \cdots & \left|T_{n, n-1}-T_{n, n}\right|
\end{array}\right)
$$

The value of every element on matrix $E$ will be either zero or nonzero. Replace the elements of nonzero with ' 1 ' in matrix $E$. Therefore, $e_{i j} \in\{0,1\}$. ' 0 ' represents that the bit of correspondent two codewords are the same and ' 1 ' represents that the bit of correspondent two codewords are different. The objective can be described as following: Select minimal number of rows from matrix $E$ to form a new matrix so as to make each sum of every column in the new matrix be greater than zero.

There follows a description of proposed shifting strategy optimization algorithm.

1. Construct a $n \times n$ matrix $P$ with $n$ patterns.

2. Based on $P$, obtain matrix $E$. Set $t=1$ and $E^{t}=E$.

3 . The size of matrix $E^{t}$ is $n^{t} \times l^{t}$. Interchange the rows of matrix $E^{t}$ to rearrange the rows according to the descent of the sum of every row. Interchange the columns of matrix $E^{t}$ to make all the elements ' 0 ' in the first row to the back of the matrix.

$E^{t}=\left(\begin{array}{ccccccc}e_{i_{1} j_{1}}^{t} & \cdots & e_{i_{1} j_{k}}^{t} & e_{i_{1} j_{k+1}}^{t} & \cdots & e_{i_{1} j_{l^{t}-1}}^{t} & e_{i_{1} j_{l^{t}}}^{t} \\ e_{i_{2} j_{1}}^{t} & \cdots & e_{i_{2} j_{k}}^{t} & e_{i_{2} j_{k+1}}^{t} & \cdots & e_{i_{2} j_{l^{t}-1}} & e_{i_{2} j_{l^{t}}} \\ \vdots & & & & & & \\ e_{i_{n^{t} j_{1}}}^{t} & \cdots & e_{i_{n^{t}} j_{k}}^{t} & e_{i_{n^{t}} j_{k+1}}^{t} & \cdots & e_{i_{n^{t}} j_{l^{t}-1}}^{t} & e_{i_{n^{t}} j_{l^{t}}}^{t}\end{array}\right)$

where $e_{i_{1} j_{1}}^{t}=\cdots=e_{i_{1} j_{k}}^{t}=1, e_{i_{1} j_{k+1}}^{t}=\cdots=e_{i_{1} j_{l} t}^{t}=0$, and $\sum_{q=1}^{l^{t}} e_{i_{1} q} \geq \sum_{q=1}^{l^{t}} e_{i_{2} q} \geq \cdots \geq \sum_{q=1}^{l^{t}} e_{i_{n} t}$.

The row number $i_{1}^{t}$ in matrix $E$ corresponding to the first row in matrix $E^{t}$ will be one optimal shifting. Record it in the shifting strategy $S_{\text {optimal }}$. If all the elements in the first row equal to 1, i.e. $\prod_{q=1}^{l^{t}} e_{i_{1} q}^{t}=1$, then stop.

4. Set $t=t+1$ and

$$
E^{t}=\left(\begin{array}{cccc}
e_{i_{2} j_{k+1}}^{t-1} & \cdots & e_{i_{2} j_{l^{t}-1}}^{t-1} & e_{i_{2} j_{l^{t}}}^{t-1} \\
\vdots & & & \\
e_{i_{n^{t-1}}^{t-1} j_{k+1}} & \cdots & e_{i_{n^{t-1} j_{l^{t-1}-1}}^{t-1}} & e_{i_{n^{t-1} j_{l^{t-1}}}^{t-1}}
\end{array}\right)
$$

Go to step 3.

$S_{\text {optimal }}$ will be one optimal shifting strategy. This solution could be used to obtain an optimal shifting strategy for arbitrary binary pattern.

\section{Experiments}

The setup of the inspection system has been detailed in [3]. The grating pattern is projected onto the target surface 
with a 30 degree inclination angle by the use of a projector. We have implemented the proposed system and tested it to reconstruct a free-form object with a period binary grating chromed on sodalime glass whose period is $1600 \mu \mathrm{m}$.

00000111110011000111001100001111

The pattern was divided into $36 \mathrm{GZs}$. Based on bitpairing codification mechanism, 4 images were enough to form unique codeword shown as following.

00000111110011000111001100001111

00111100000111110011000111001100

10011000111001100001111000001111

11110011000111001100001111000001

However, at least 6 images were needed if not adopting bit-pairing encoding mechanism.

00000111110011000111001100001111
00111100000111110011000111001100
01100001111000001111100110001110
10011000011110000011111001100011
10001110011000011110000011111001
10011000111001100001111000001111

In the calibration phase, we projected the fringe pattern onto a sheet of white paper, which served as the reference plane. By this step, a codeword for each position on the reference plane was transferred from the corresponding position on the grating plane. Depth determination processing could thus be transferred from over the grating-andimage plane-pair to over the reference-and-image planepair. The image sequence for the reference plane is shown in Fig. 2(a). In the operation phase, we projected the same fringe pattern onto a free-form object. With four shiftings of the pattern and imagings of the illuminated object, we established also a codeword for each point on the inspected surface. To put it simply, the codeword is the sequence of "1"s and " 0 "s in the image sequence (shown in Fig. 2(b)) at every image position. With calibration and operation image sequence, correspondence between reference plane and object can be easily established. With the correspondences, 3D position or depth disparity could be estimated for each point of the inspected surface through triangulation. Fig. 2(c) shows the 3D reconstruction of the inspected surface.

\section{Conclusion and Future Work}

We have described an approach for $3 \mathrm{D}$ reconstruction, that is based upon projecting a binary grating with several spatial shiftings of it. To reduce the required image number so as to improve the reconstruction speed, we have presented a bit-pairing codification mechanism. A solution to shifting strategy optimization based on elementary matrix operations has been proposed that is applicable to any given binary patterns. Experiments on free-form objects demonstrated that image number could be reduced with the ap-

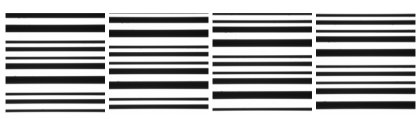

(a) Image sequence of the reference plane in the calibration phase.

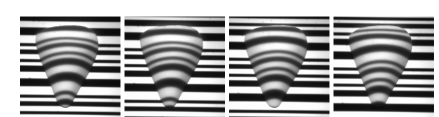

(b) Image sequence of the inspected surface in the operation phase.

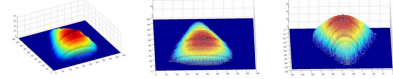

(c) 3D reconstruction of the inspected surface, as observed from different angles

\section{Figure 2. Experiment on free-form object with bit-pairing codification mechanism.}

proach. In the future, we will further study how to design the optimal pattern while adopting bit-pairing codification to minimize the required image number.

\section{References}

[1] M. D. Altschuler, B. R. Altschuler, J. T. Dijak, L. A. Tamburino, and B. Woolford. Robot vision by encoded light beams. In T. Kanade, editor, Three-Dimensional Machine Vision, pages 53-71. Kluwer Academic, Dordrecht, 1987.

[2] M. D. Altschuler, B. R. Altschuler, and J. Taboada. Laser electro-optic system for rapid three-dimensional (3-d) topographic mapping of surfaces. Optical Engineering, 20(6):953-961, 1981

[3] J. Cheng, R. Chung, E. Lam, K. Fung, F. Wang, and W. Leung. Three-dimensional reconstruction of wafer solder bumps using binary pattern projection. volume 5679 of Proc. SPIE, pages 44-52, 2005.

[4] O. Hall-Holt and S. Rusinkiewicz. stripe boundary codes for real-time structured-light range scanning of moving objects. ICCV, pages 359-366, 2001.

[5] P. S. Huang, C. P. Zhang, and F. P. Chiang. High speed 3-d shape measurement based on digital fringe projection. Optical Engineering, 42(1):163-168, 2003.

[6] S. Inokuchi, K. Sato, and F. Matsuda. Range-imaging for 3-d object recognition. ICPR, pages 806-808, 1984.

[7] S. Kim, Y. Choi, and J. Oh. Three-dimensional profile measurement of fine object by phase-shifting moiré interferomety. volume 2909 of Proc. SPIE, pages 28-36, 1997.

[8] J. L. Posdamer and M. Altschuler. Surface measurement by space-encoded projected beam systems. Computer Graphics and Image Processing , 18(1):1-17, 1982. 